\title{
Deep Subwavelength Light Confinement in Disordered Bismuth Nanorods as a Linearly Thermal-Tunable Metamaterial
}

\author{
Mahmut Can Soydan,* Amir Ghobadi, Deniz Umut Yildirim, Vakur Behcet Erturk, \\ and Ekmel Ozbay
}

Materials with a tunable optical response that can be controllably tailored using external stimuli excitation have undergone considerable research effort for the development of active optical devices, such as thermo-optical modulators. Although bismuth (Bi) nanodots, embedded into glass matrices, have been proven to have a thermo-optical response, the recyclability of the structure in solid-liquid phase transitions is a major challenge. Herein, a facile and lithography-free fabrication method is proposed to realize densely packed standalone Bi nanorods (NRs), with deep subwavelength gaps and a resonance at the

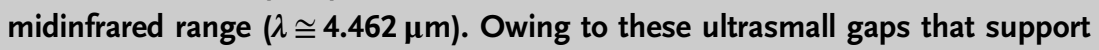
lossy Mie-like resonances, strong field confinement is achieved, and the resonance wavelength exhibits great sensitivity to temperature, as the thermal sensitivity reaches as high as $1.0316 \mathrm{~nm}^{\circ} \mathrm{C}^{-1}$. This operation is conducted in the moderate temperature interval of $25-85^{\circ} \mathrm{C}$, which is far from the melting point of Bi. Overall, our simple, robust, and high-performance device is highly promising for realizing optical switches, thermo-optic modulators, and infrared camouflage.

dimensions and, therefore, functional optical devices require complex fabrication routes, such as electron beam lithography (EBL). Moreover, dynamic control of these optical properties is another issue that has attracted much attention in recent years. To attain the possibility of real-time tuning of the EM response of the metadevices, dynamically tunable scenarios are needed. Several techniques, such as electrical, ${ }^{[4]}$ optical, ${ }^{[5]}$ and thermal ${ }^{[6]}$ tuning, have been proposed in the literature to realize active tunable metamaterials.

The class of thermally tunable metamaterial perfect absorbers (MPAs) are emerging as promising candidates in the applications of optical modulators and infrared camouflage. ${ }^{[7,8]}$ Their operation principle lies in the thermo-optical effect of the change in the optical constants of a material with temperature, ${ }^{[9]}$ which is

Efficient confinement and harvesting of the electromagnetic (EM) radiation is one of the most vigorously studied research areas under metamaterials, due to their vast variety of applications. ${ }^{[1-3]}$ These attractive properties are, nevertheless, acquired by the periodic arrangement of subwavelength material

M. C. Soydan, A. Ghobadi, D. U. Yildirim, Prof. E. Ozbay

NANOTAM-Nanotechnology Research Center

Bilkent University

06800 Ankara, Turkey

E-mail: soydan@ee.bilkent.edu.tr

M. C. Soydan, A. Ghobadi, D. U. Yildirim, Prof. V. B. Erturk, Prof. E. Ozbay Department of Electrical and Electronics Engineering

Bilkent University

06800 Ankara, Turkey

Prof. E. Ozbay

Department of Physics

Bilkent University

06800 Ankara, Turkey

Prof. E. Ozbay

UNAM-Institute of Materials Science and Nanotechnology

Bilkent University

06800 Ankara, Turkey

The ORCID identification number(s) for the author(s) of this article can be found under https://doi.org/10.1002/pssr.202000066.

DOI: 10.1002/pssr.202000066 prominent in the phase-change materials, such as GeSbTe (GST) and vanadium dioxide $\left(\mathrm{VO}_{2}\right)$ as well as amorphous silicon. ${ }^{[10-12]}$ Thermally tunable MPAs need to fulfill the requirements of high sensitivity, spectrally selectivity, and low-power dissipation. This makes low-melting-point metals, such as bismuth (Bi), zinc $(\mathrm{Zn})$, gallium $(\mathrm{Ga})$, lead $(\mathrm{Pb})$, and tin $(\mathrm{Sn})$, candidates for such applications. ${ }^{[13]}$ Therefore, taking all of this into account, the large-scale realization of MPAs is crucial for their mass-production with high-throughput as well as repeatability. ${ }^{[14]}$ Zhao et al. reported a linearly thermal-tunable ultra-narrowband MPA based on four-nanorod (NR)-coupled amorphous silicon with a sensitivity of $0.08 \mathrm{~nm}^{\circ} \mathrm{C}^{-1}$. [11] They revealed that the shift in the resonance wavelength from 1164 to $1172 \mathrm{~nm}$ proportionally increasing temperature depends on the change of the optical constants. However, the proposed design is not large-scale compatible as well as findings are numerical based.

Among the aforementioned low-melting-point materials cited herein, $\mathrm{Bi}$ is a low-toxicity, low-cost, earth-abundant heavy element with a melting point of $271.3^{\circ} \mathrm{C} .{ }^{[15]}$ In its solid bulk state, Bi presents semimetallic properties. The real part of the dielectric permittivity of solid Bi from ultraviolet to near-infrared spectral ranges presents negative values. ${ }^{[16,17]}$ In this spectral range, the liquid phase $\mathrm{Bi}$ is a Drude metal with strong losses, ${ }^{[18]}$ and thus a "lossy" plasmonic material. Bi nanoparticles (NPs), embedded 
inside stable host matrices (such as germanate glass or amorphous aluminum oxide), have been proven to have a thermally tunable optical response exhibiting a transmission-temperature hysteresis loops. ${ }^{[19,20]}$ However, the structural deformation in multiple melting-solidification could significantly hamper the recyclability of this design. Moreover, the necessity of melting and solidifying Bi still requires relatively large thermal budgets, and consequently slow transitions. ${ }^{[19]}$ In this work, we experimentally realized a $\mathrm{Bi}$ nanostructure-based linear thermaltunable MPA design in the midinfrared (MIR) regime, within a temperature window much below the melting point. The design is a metal-metal (MM) configuration that consists of a bottom thick Bi layer, acting as a mirror, and a top layer of densely packed Bi NRs, operating as a light trapping scaffold. Considering the fact that EBL is a large-scale incompatible route and fabrication of high aspect ratio structures are challenging, the NRs are fabricated by adopting the oblique-angle deposition technique ${ }^{[21,22]}$ in a complete lithography-free and easily repeatable route. Experimental findings demonstrate an MIR MPA with a peak absorption at $4.46 \mu \mathrm{m}$. The evolution of the resonance spectral position, upon temperature tuning in the range of $25-85^{\circ} \mathrm{C}$, proves the strong thermo-optical response of the structure with a sensitivity as high as $1.0316 \mathrm{~nm}^{\circ} \mathrm{C}^{-1}$. This unprecedented highly sensitive functionality originates from the formation of strong light confinement in deep subwavelength gaps between NRs that triggers a strong light-matter interaction. Thus, the choice of material and the elegant fabrication route provide thermo-optic tuning in temperatures much below the melting point with no need to material phase change. Overall, this work unveils the potential of solid Bi in thermo-optical applications such as switching, modulators, and infrared camouflage.

In the first step, numerical simulations are conducted to scrutinize the impact of geometrical parameters of NRs on the absorption capacity of the MM MPA. For this purpose, a commercial finite-difference time-domain (FDTD) software package (Lumerical FDTD Solutions) is used. The dielectric constant of Bi was modeled and extracted through J.A. Woollam Co. IR-Vase Mark II Ellipsometer, as explained in our previous study. ${ }^{[22]} \mathrm{We}$ simply assume a periodic arrangement of a unit cell, as shown in Figure 1a. The extracted real and imaginary parts of the dielectric constant of $\mathrm{Bi}$ are shown in Figure $1 \mathrm{~b}$. The bottom Bi layer is only a mirror that should be optically thick enough to block light transmission. Thus, the dominant geometries are the top NRs' height $(H)$ and width $(W)$. The periodicity $(P)$ is fixed at $200 \mathrm{~nm}$ and a sweep is performed on the aforementioned parameters. The contour plot in Figure 1c shows the impact of $W$ on the absorption response of Bi-based MPA, whereas $H$ is fixed at $300 \mathrm{~nm}$. As clearly revealed from this panel, at small widths, no significant light-matter interaction is acquired. However, for $W$ values larger than $\approx 140 \mathrm{~nm}$, a narrow but near unity absorption can be achieved. Larger width, i.e., smaller gap, substantiates the absorption strength and bandwidth of the design. Thus, to have an MIR-responsive MPA, deep subwavelength gap formation is essential. A similar sweep is performed on parameter $H$, whereas the $W$ is fixed at the optimal value of $180 \mathrm{~nm}$. As shown in Figure $1 d$, near-perfect absorption in this MPA requires a height larger than $\approx 200 \mathrm{~nm}$. As we move to larger $H$ values, the absorption bandwidth enhances. Considering the targeted tunable MPA (a)

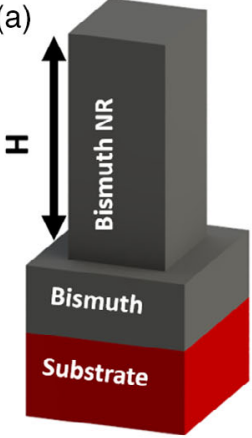

(c)

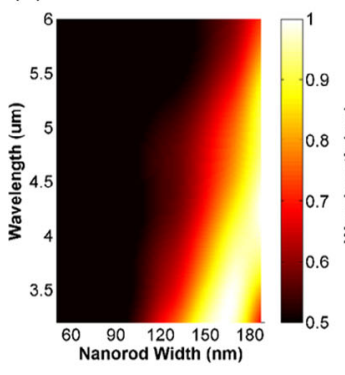

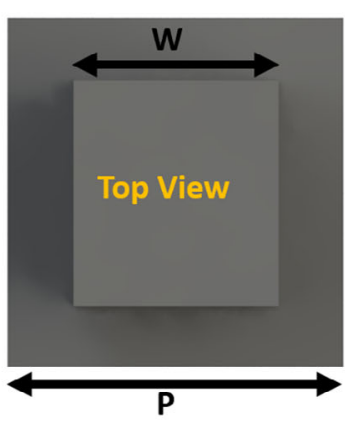

(d)

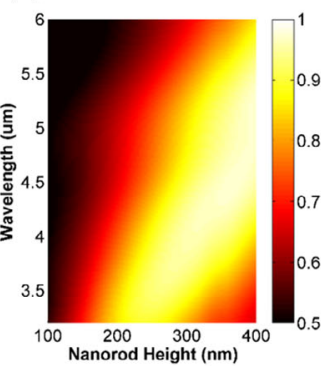

(b)

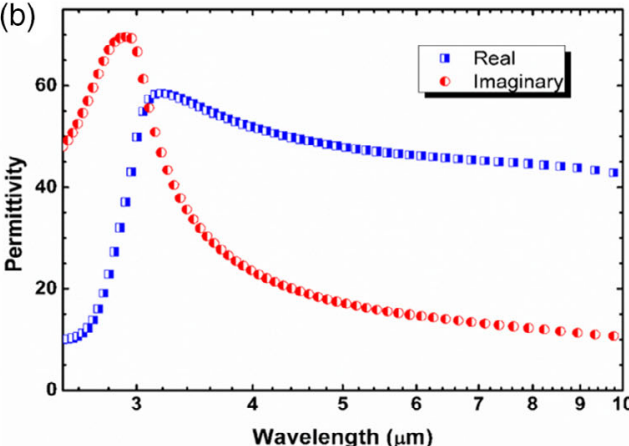

(e)

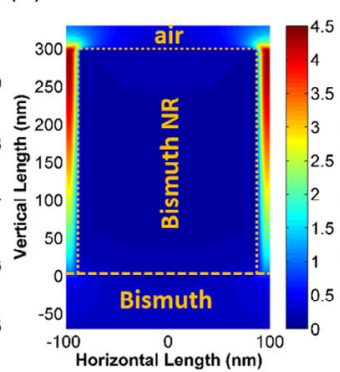

(f)

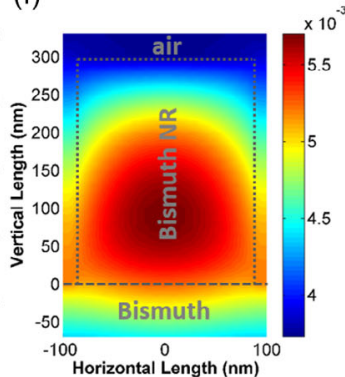

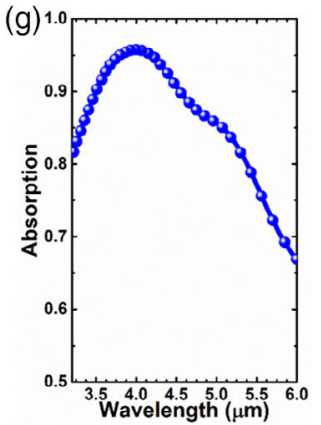

(h)

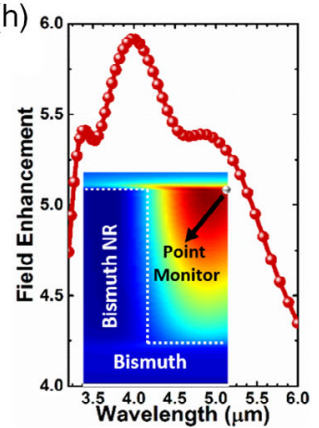

Figure 1. a) Schematic illustration of the MM MPA unit cell and its top view. b) Optical permittivity of Bi used in the simulations. The contour plots showing the evolution of absorption response as a function of c) NRs' width (W), d) height (H), e) E-field, and f) $H$-field distributions across the MM cavity at the resonance frequency. The color bar unit for E-field is $\mathrm{Vm}^{-1}$ and for $\mathrm{H}$-field it is $\mathrm{Am}{ }^{-1}$. g) The absorption profile of the MM design with the optimal dimensions of $W=180 \mathrm{~nm}$, and $H=300 \mathrm{~nm}$, and corresponding $\mathrm{h}$ ) near-field enhancement at the mid of gap region. The inset shows the position of the point monitor that was used for E-field probing. 
application that requires a narrow but near-unity light absorption, the optimal value for $H$ can be considered as $\approx 300 \mathrm{~nm}$. It can be added that sweeping the parameter of $P$ corresponds to sweeping of $W$ because filling fraction of the $\mathrm{Bi}$ metal to the air dielectric is essential on the response of the structure. Therefore, effect of $P$ on absorption based on numerical simulation is not shared and value of $200 \mathrm{~nm}$ is selected based on fabrication findings.

To gain an insight into the origin of this response, electric $(E)$ and magnetic $(H)$ field distributions have been extracted for this MPA, as shown in Figure 1e,f, respectively. These panels demonstrate that the obtained near-unity resonance absorption stems from the simultaneous coupling of electric and magnetic dipole resonances. This efficient coupling, in turn, leads to the creation of strong E-fields in the gap regions. As shown in Figure 1g,h, the absorption profile of the Bi-based MPA is in close relation with the near-field enhancement spectral profile. When taking all of the aforementioned results into account, a Bi-based MIRresponsive MPA can be theoretically accomplished. However, this necessitates densely packed Bi NRs with moderately large aspect ratios (ARs) which is essentially a challenging architecture for common nanofabrication tools including EBL. To fulfill this goal, we have adopted a large-scale compatible route with no material restriction to realize high ARs, and densely packed Bi NRs.

For this aim, the oblique-angle deposition technique ${ }^{[21]}$ was used. This method exploits the inherent line-of-sight coating principle of physical vapor deposition (PVD) systems along with shadowing to achieve quasi-3D nanosized columnar fills (NRs) with an intrinsic tilt and porosity. In this fabrication route, the sample is placed in a near vertical orientation (with an approximate angle of $\theta<10^{\circ}$ ) in the thermal evaporation tool. The mechanism responsible for the creation of $\mathrm{Bi}$ nanostructures is schematically shown in Figure 2a. As shown in this panel, the formation of these nanoislands is promoted via the shadowing effect of the NRs. At the first moments of deposition, primary evaporated Bi molecules stick to the sample surface first, and obstruct the secondary evaporated molecules to reach to the region shadowed by them. And therefore, tightly located Bi nanoislands are formed. As deposition continues, the smaller islands are shadowed by the larger ones and, consequently, Bi NRs with larger spacing occur. These facts can be easily extracted from a (a)

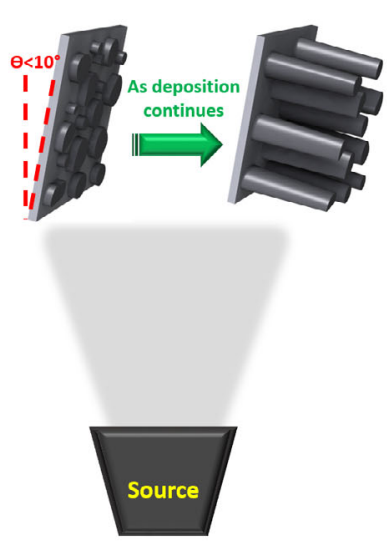

(e)

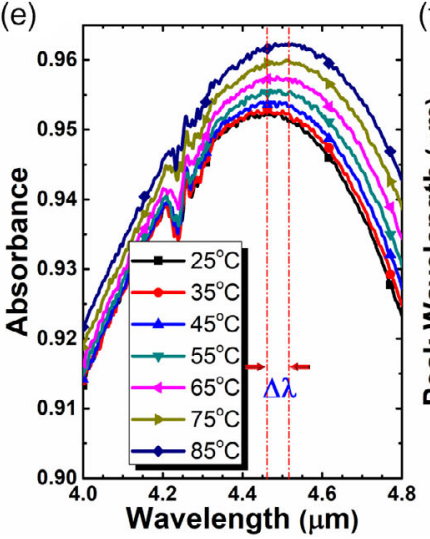

(b)

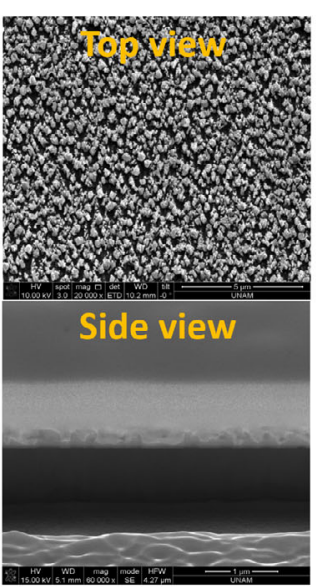

(c)

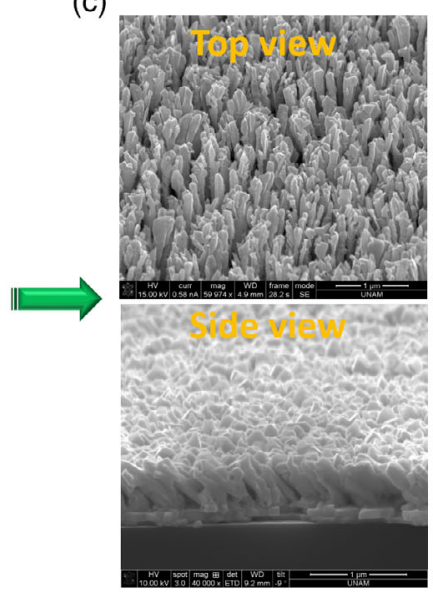

(g)

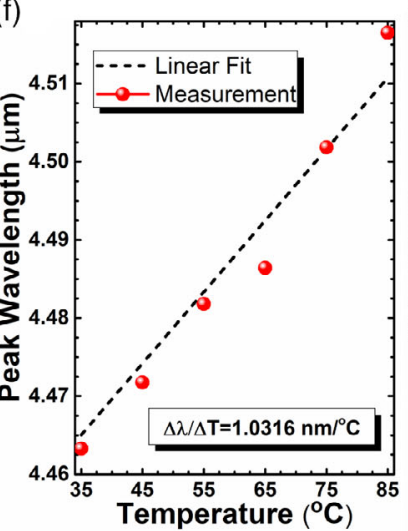

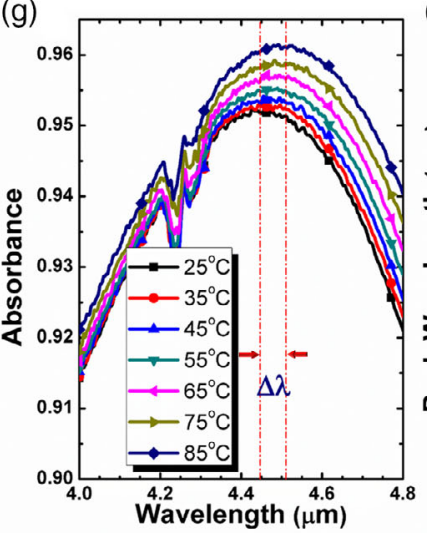

(d)

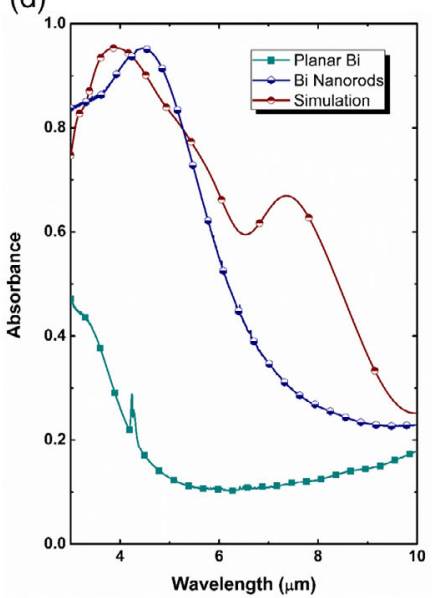

(h)

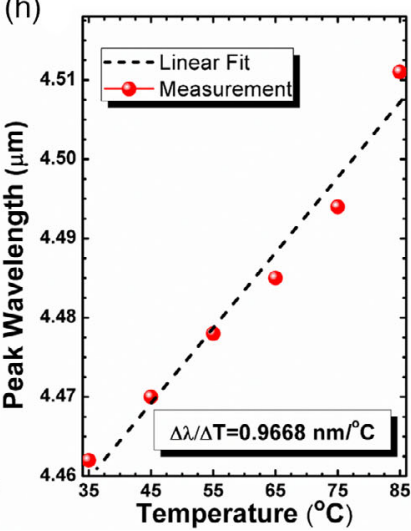

Figure 2. a) Schematic illustration of line-of-sight coating in a PVD system and the formation of tilted nanowires. Top-view SEM micrograph and crosssectional FIB image of the sample b) during the early moments of the fabrication that promotes the formation of Bi nanoislands, and c) after a specified longer time leads to densely packed Bi NRs. d) Experimentally measured and simulated absorption spectra for the proposed MM MPA device, and experimental absorption spectrum of a bare planar sample. e) The spectral absorption peak resonance wavelength change of the fabricated device as a function of temperature, and f) the estimated thermal-optical sensitivity through the linear fitting of the data. g,h) Repeated measurements for recyclability test. 
top-view scanning electron microscope (SEM) and the side-view focused ion beam (FIB) images of the thin and thick Bi NR samples, as shown in Figure 2b,c, respectively. As shown in these panels, for the fabrication of this MPA, first a $150 \mathrm{~nm}$-thick planar Bi back-reflector layer and then Bi NRs of a length of $250 \mathrm{~nm}$ were coated in thermal evaporation equipment with an oblique angle deposition approach to obtain MIR response based on simulation findings. Preferential deposition at random nucleation sites, which are formed during the initial stages of deposition, makes the overall design a random structure. Therefore, discussing certain values of orientation angle, length, and radius of the NRs is out of the question. Various factors such as the placement angle of the sample to the vertical direction (i.e., $\theta$ ), deposition rate and time, and the distance between the source material and sample have an important effect on the geometrical parameters of the self-aligned NRs. However, definite effects of these factors can only be identified under complete control experiments. The random formation of such nanoislands and NRs is demonstrated in SEM and FIB images (see Figure 2b,c). It is observed that as the deposition time and rate is increased, NRs become longer and densely packed.

Next, the sample was optically characterized using Fourier transform infrared spectroscopy (FTIR) technique. The exactly measured absorbance spectrum of the fabricated proposed device, measured at room temperature, is shown in Figure 2d, in which an absorption resonance peak around $4.462 \mu \mathrm{m}$ is discernible. There are two main underlying physical reasons in achieving this resonance. Our designed architecture provides strong light-matter interaction, due to the highly diffracting/ scattering and trapping nature of the top-layer scaffold. ${ }^{[20,23,24]}$ The trapped radiation is then coupled to $\mathrm{Bi}$ and harvested there because of the lossy dielectric nature of $\mathrm{Bi}$ in the MIR regime, with a positive real part of permittivity and small imaginary part of permittivity. ${ }^{[17,24-27]}$ This particular dispersion of $\mathrm{Bi}$ in the MIR spectral range suggests a Mie-like resonance with a relatively low Q-factor, ${ }^{[25]}$ which is in line with our finding. We compared the spectral response of our proposed device to that of a design that only uses a planar layer of $\mathrm{Bi}$ with a thickness of $150 \mathrm{~nm}$. From Figure 2d, it can be seen that planar design offers no spectral selectivity. Therefore, creating NRs with an oblique deposition without changing material introduces a resonance and increases overall absorption of the structure in the MIR regime. We also compared the numerical and experimental results. Figure $2 \mathrm{~d}$ shows that absorbance envelope of both results is in great harmony with tiny differences. For example, resonance wavelength around $3.9 \mu \mathrm{m}$ in simulation shifts to $4.462 \mu \mathrm{m}$. However, it should be noted that great fit between them is not expected because it is unfeasible to simulate a completely random design. As the next step, we scrutinized the thermo-optical response of our proposed design by means of measuring its absorbance spectrum at different temperatures. The temperature of the sample was controlled using a homemade thermoelectric Peltier element which uses 5310 temperature controller of "arroyo instruments." Figure 2e shows the absorbance spectrum around the resonance wavelength for swept temperature values between 25 and $85^{\circ} \mathrm{C}$. The red shift of the resonance wavelength is clearly observed with increasing temperature. The small ripples on the experimentally attained data obstruct calculating the precise resonance wavelength.
Therefore, we applied a seventh-degree polynomial fit onto the experimental data to find the exact position of the peak wavelength. The extracted resonance wavelengths, which are shown in Figure 2f, linearly shift from 4.462 to $4.517 \mu \mathrm{m}$ with increasing temperature. A linear fit is applied to find the thermo-optical sensitivity of the sample, which is calculated with the formula of $S=\Delta \lambda / \Delta T$. Thermo-optical sensitivity coefficient, in other words, the slope of the linearly fitted line, is calculated as $S=1.0316 \mathrm{~nm}^{\circ} \mathrm{C}^{-1}$ as shown in Figure $2 \mathrm{~g}$, which is on par with and even higher than the other reported experimental and numerical values that are obtained with different geometries and other materials. ${ }^{[11,28]}$ Zhao et al. achieved a linearly thermaltunable MPA with a sensitivity of $0.08 \mathrm{~nm}^{\circ} \mathrm{C}^{-1}$ which has resonance at $1064 \mathrm{~nm} .{ }^{[11]}$ The sensitivity of our proposed design is nearly 13 times greater than they reported. If we analyze the ratio of sensitivity to resonance wavelength to interpret resonance shift with respect to resonance wavelength, densely packed Bi NRs still have 3 times higher sensitivity. Due to the strong light confinement in high AR gap volumes, the small change in the refractive index of $\mathrm{Bi}$ is intensified and large $S$ values are obtained. It should also be noted that while their work is based on numerical simulations, our findings are experimental results. Moreover, the Bi dielectric can be modelled as sum of Drude and Lorentz oscillators, ${ }^{[27]}$ in which the amplitude of plasma oscillation is related to the carrier concentration. The dispersion of the energy band of the Bi can lead to the change of the conductivity. Therefore, the thermo-optic behavior of the design can also be related to the dispersion of the energy band of Bi NRs, similar to the other materials. ${ }^{[2-31]}$ Lastly, to ensure the recyclability of the design, a second measurement is conducted, and the $S$ is extracted for this case, as shown in Figure $2 \mathrm{~h}$. Based on this data, a quite similar performance is observed $\left(S=0.9668 \mathrm{~nm}^{\circ} \mathrm{C}^{-1}\right)$.

Overall, strong field confinement in deep subwavelength gaps of high AR Bi NR arrays triggered a significantly accentuated thermo-optical sensitivity, in a temperature range much below the melting point of the material, i.e., an intermediate solidliquid phase. Despite the small tuning of the Bi permittivity in this state, the formation of intense hot spots maximizes the sensitivity of resonance frequency to small permittivity changes. To analyze this from a different perspective, realizing such deep subwavelength gaps is crucial for increasing the filling factor of $\mathrm{Bi}$ in the Bi-air composite so that the effective medium is influenced more by the small changes in the refractive index of $\mathrm{Bi}$ with temperature. Our method, then, not only results in a large-scale compatible device but also it creates ultra-narrow gaps and a high AR scaffold, which is very strenuous to be achieved by EBL. Using this technique, a 4 " Si wafer can be uniformly coated with high AR Bi NRs. ${ }^{[22]}$ In addition, the response of this device does not stem from the melting-solidification transition that deforms the integrity of the design, so it provides a stable and extendable platform for efficient thermal-optical device designs such as optical modulators, optical switches, and optical modulators. ${ }^{[7,8]}$

In conclusion, we analyzed and experimentally confirmed the unprecedented thermo-optical sensitivity of an MM MPA that is entirely made of $\mathrm{Bi}$. To fabricate this sample, oblique angle deposition is utilized not only as a lithography-free and a large-scale compatible route, but also to achieve a scaffold that efficiently 
confines and harvests the MIR radiation with a spectrally selective resonant response. We verified the existence of such a resonance at $4.462 \mu \mathrm{m}$, when the sample is measured at room temperature. This resonance showed great sensitivity to the external temperature altering, as the thermo-optical sensitivity reached a significant value of $1.0316 \mathrm{~nm}^{\circ} \mathrm{C}^{-1}$. The mechanism behind this spectrally selective optical response, in other words, the resonance, and its high sensitivity to the moderate heating are attributed to the strong field confinement in deep subwavelength gap regions. This work highlights the impact of the optical response of an MPA design on its sensitivity, in which a proper field confinement can provide a linearly thermaltunable absorber, without the need for solid-liquid phase transitions. Overall, our simple yet robust, and easy-to-fabricate device is highly promising for the areas of thermo-optic modulators, switches, and infrared camouflage.

\section{Experimental Section}

Fabrication of Bi NRs: A diced silicon wafer in the size of $(2 \mathrm{~cm} \times 2 \mathrm{~cm})$ was used as the substrate of the sample. This square shaped silicon substrate was cleaned using sonication bath with acetone for 20 min and isopropanol for $10 \mathrm{~min}$, respectively, and then dried with $\mathrm{N}_{2}$ gas flow. Surface purity of the sample was checked using optical microscope to be ensured that it was totally clean for the rest of the process. The two-step coating of the designed structure was realized using MIDAS PVD 3T model thermal evaporator system. The $150 \mathrm{~nm}$-thick planar back-reflector layer was deposited in the normal usage procedure, i.e., sample is placed to the sample holder at the top of the vacuum chamber. This layer was deposited at an initial chamber pressure of $3 \times 10^{-6}$ Torr (reached to the $4 \times 10^{-6}$ Torr during evaporation) with an evaporation rate of $3 \AA \mathrm{s}^{-1}$. Next, the densely packed $\mathrm{Bi}$ NRs were created adapting oblique-angle deposition method to self-shadowing approach as described in the article. In this step, the sample was fixed onto a sample holder that we prepared to hold the sample upright, as shown in Figure 2a. The large vacuum chamber of MIDAS PVD 3T lets us to implement our sample holder. This evaporation process was completed at the same chamber pressure, but with an evaporation rate of $9 \AA \mathrm{s}^{-1}$ to intensify the effectivity of the shadowing effect.

Optical Characterization: To optically characterize the sample, its reflection spectrum was measured using the FTIR technique (BrukerVertex 70v, Hyperion microscope). The instrument utilized a MIR source, $\mathrm{KBr}$ beam splitter, and nitrogen-cooled Mercury Cadmium Telluride (MCT-D313) detector. A $125 \mathrm{~nm}$ gold-coated substrate was used as a reference. From the reflection spectrum, the absorption was calculated using the formula, $A=1-R$, in which the transmission was neglected due to the thick back metal layer. To precisely control the temperature, we placed a homemade thermoelectric Peltier element, which used 5310 model of temperature controller of "arroyo instruments," onto the stage of Hyperion microscope, and left the sample onto it. Excellent stability and auto-tune proportional-integral-derivative (PID) calculation properties of the temperature controller enabled us to easily and precisely set up the temperature. After the temperature was increased to next level (i.e., $10^{\circ} \mathrm{C}$ higher), $5 \mathrm{~min}$ was waited to uniformly distribute and stabilize the temperature all over the sample before the reflection measurement was taken using FTIR to have more reliable results. This process was repeated from 25 to $85^{\circ} \mathrm{C}$ with $10^{\circ} \mathrm{C}$ increments.

\section{Acknowledgements}

The authors acknowledge financial support from Scientific and Technological Research Council of Turkey (TUBITAK) and DPT-HAMIT under the Project Nos. 113E331, 114E374, and 115F560. One of the authors (E.O.) also acknowledges partial support from the Turkish Academy of Sciences (TUBA).

\section{Conflict of Interest}

The authors declare no conflict of interest.

\section{Keywords}

bismuth, lithography-free, metamaterials, thermally-tunable

Received: February 9, 2020

Revised: April 13, 2020

Published online: April 29, 2020

[1] K. Chaudhuri, M. Alhabeb, Z. Wang, V. M. Shalaev, Y. Gogotsi, A. Boltasseva, ACS Photonics 2018, 5, 1115.

[2] M. Zhang, X. X. Wang, W. Q. Cao, J. Yuan, M. S. Cao, Adv. Opt. Mater. 2019, 7, 1900689

[3] M. S. Cao, X. X. Wang, M. Zhang, J. C. Shu, W. Q. Cao, H. J. Yang, X. Y. Fang, J. Yuan, Adv. Funct. Mater. 2019, 29, 1807398.

[4] Z. Zhu, P. G. Evans, R. F. Haglund, J. G. Valentine, Nano Lett. 2017, $17,4881$.

[5] M. Abb, P. Albella, J. Aizpurua, O. L. Muskens, Nano Lett. 2011, 11, 2457.

[6] K. Appavoo, D. Y. Lei, Y. Sonnefraud, B. Wang, S. T. Pantelides, S. A. Maier, R. F. Haglund, Nano Lett. 2012, 12, 780

[7] S. Chandra, D. Franklin, J. Cozart, A. Safaei, D. Chanda, ACS Photonics 2018, 5, 4513.

[8] X. Tian, Z. Y. Li, Plasmonics 2018, 13, 1393.

[9] G. Coppola, Opt. Eng. 2011, 50, 071112.

[10] K. Sun, C. A. Riedel, A. Urbani, M. Simeoni, S. Mengali, M. Zalkovskij, B. Bilenberg, C. H. De Groot, O. L. Muskens, ACS Photonics 2018, 5, 2280.

[11] L. Zhao, X. Yang, Q. Niu, Z. He, S. Dong, Opt. Lett. 2019, 44, 3885

[12] T. Cao, X. Zhang, W. Dong, L. Lu, X. Zhou, X. Zhuang, J. Deng, X. Cheng, G. Li, R. E. Simpson, Adv. Opt. Mater. 2018, 6, 1800169.

[13] G. V. Naik, V. M. Shalaev, A. Boltasseva, Adv. Mater. 2013 , $25,3264$.

[14] A. Ghobadi, H. Hajian, B. Butun, E. Ozbay, ACS Photonics 2018, 5,4203

[15] A. Ghobadi, H. Hajian, M. Gokbayrak, B. Butun, E. Ozbay, Nanophotonics 2019, 8, 823 .

[16] O. Hunderi, J. Phys. 1975, 5, 2214.

[17] J. Toudert, R. Serna, Opt. Mater. Express 2017, 7, 2299.

[18] S. Dogel, D. Nattland, W. Freyland, Phys. Rev. B, Condens. Matter Mater. Phys. 2005, 72, 085403.

[19] M. Jiménez De Castro, R. Serna, J. Toudert, J. M. Fernández Navarro, E. Haro-Poniatowski, Ceram. Int. 2015, 41, 8216

[20] J. Toudert, R. Serna, M. Jiménez De Castro, J. Phys. Chem. C 2012, 116, 20530.

[21] C. Grüner, S. Liedtke, J. Bauer, S. G. Mayr, B. Rauschenbach, ACS Appl. Nano Mater. 2018, 1, 1370.

[22] M. C. Soydan, A. Ghobadi, D. U. Yildirim, E. S. Duman, A. Bek, V. B. Erturk, E. Ozbay, Adv. Opt. Mater. 2020, 8, 1901203.

[23] D. Ji, A. Cheney, N. Zhang, H. Song, J. Gao, X. Zeng, H. Hu, S. Jiang, Z. Yu, Q. Gan, Adv. Opt. Mater. 2017, 5.

[24] J. Toudert, R. Serna, M. G. Pardo, N. Ramos, R. J. Peláez, B. Maté, Opt. Express 2018, 26, 34043. 
[25] J. Toudert, R. Serna, I. Camps, J. Wojcik, P. Mascher, E. Rebollar, T. A. Ezquerra, J. Phys. Chem. C 2017, 121, 3511.

[26] A. P. Lenham, D. M. Treherne, R. J. Metcalfe, J. Opt. Soc. Am. 1965, $55,1072$.

[27] J. Toudert, R. Serna, Opt. Mater. Express 2016, 6, 2434.

[28] W. Lewandowski, M. Fruhnert, J. Mieczkowski, C. Rockstuhl, E. Górecka, Nat. Commun. 2015, 6, 6590.
[29] X. Y. Fang, X. X. Yu, H. M. Zheng, H. B. Jin, L. Wang, M. S. Cao, Phys. Lett. A 2015, 379, 2245.

[30] Y. H. Jia, P. Gong, S. L. Li, W. D. Ma, X. Y. Fang, Y. Y. Yang, M. S. Cao, Phys. Lett. A, Gen. At. Solid State Phys. 2020, 384, 126106.

[31] Y. J. Li, S. L. Li, P. Gong, Y. L. Li, M. S. Cao, X. Y. Fang, Physica E, Low-Dimensional Syst. Nanostruct. 2018, 98, 191. 